“C 2018 IEEE. Personal use of this material is permitted. Permission from IEEE must be obtained for all other uses, in any current or future media, including reprinting/republishing this material for advertising or promotional purposes, creating new collective works, for resale or redistribution to servers or lists, or reuse of any copyrighted component of this work in other works." 


\title{
Feature Analysis for Discrimination of Motor Unit Action Potentials
}

\author{
Thuy T. Pham ${ }^{* 12}$, Diep N. Nguyen ${ }^{1}$, Eryk Dutkiewicz ${ }^{1}$, \\ Alistair L. McEwan ${ }^{2}$, and Philip H.W. Leong ${ }^{2}$, Andrew J. Fuglevand ${ }^{3}$, \\ ${ }^{1}$ Faculty of Engineering and IT, University of Technology Sydney, NSW, Australia. \\ ${ }^{2}$ Department of Electrical and Information Engineering, University of Sydney, NSW, Australia. \\ ${ }^{3}$ Department of Physiology, University of Arizona, AZ, USA.
}

\begin{abstract}
In electrophysiological signal processing for intramuscular electromyography data (nEMG), single motor unit activity is of great interest. The changes of action potential (MUAP) morphology, motor unit (MU) activation, and recruitment provide the most informative part to study the nature causality in neuromuscular disorders. In practice, for a single nEMG recording, more than one motor unit activities (in the surrounding area of a needle electrode) are usually collected. Such a fact makes the MUAP discrimination that separates single unit activities a crucial task. Most neurology laboratories worldwide still recruit specialists who spend hours to manually or semi-automatically sort MUAPs. From a machine learning perspective, this task is analogous to the clustering-based classification problem in which the number of classes and other class information are unfortunately missing. In this paper, we present a feature analysis strategy to help better utilize unsupervised (i.e., totally automated) methods for MUAP discrimination. To that end, we extract a large pool of features from each MUAP. Then we select the top ranked candidates using clusterability scores as selection criteria. We found spectrograms of wavelet decomposition as a top-ranking feature, highly correlated to the motor unit reference and was more separable than existing features. Using a correlation-based clustering technique, we demonstrate the sorting performance with this feature set. Compared with the reference produced by human experts, our method obtained a comparable result (e.g., equivalent number of classes was found, identical MUAP morphology in each pair of corresponding MU class, and similar histograms of MUs). Taking the manual labels as references, our method got a much higher sensitivity and accuracy than the compared unsupervised sorting method. We obtained a similar result in MUAP classification to the reference.
\end{abstract}

Index Terms-Spike sorting, feature learning

\section{INTRODUCTION}

Motor unit activity analysis provides crucial information towards diagnosis and treatment of neuromuscular disorders. In intramuscular electromyography data, when recording small voluntary contractions with a needle electrode, the electrical signal obtained is often a combination of more than one motor unit (MU) from the surrounding area of the needle tip. Therefore, a motor unit action potential (MUAP) consists of several muscle fiber action potentials (MFAPs) within the anatomical MU.

To obtain the changes of MUAP morphology, MU activation, and $\mathrm{MU}$ recruitment that yield valuable information, one would require activities from a single MU. Neuropathic conditions occur with decreased recruitment whereas myopathic conditions happen with MUAP morphology changes. As an example, a MUAP examination can confirm myopathic

*: correspondence thuy.pham@uts.edu.au. Faculty of Engineering and IT, University of Technology Sydney, NSW, Australia conditions and identify the differential to find an appropriate biopsy site [1]. For each recording, most neurology laboratories employ experts who spend hours to classify action potentials ("spikes") using commercial software tools (e.g., Spike2 [2], Cerebus [3]) . This de-facto standard practice relies on human-based assessment which is subjective (hence prone to mistake/errors of the expert) and time-consuming. An unsupervised/automated classification method is hence very much desirable.

A spike discrimination procedure involves three basic phases: spike detection, feature extraction, and spike clustering. Spike detection often involves aligning spikes to a common temporal point. The feature extraction phase provides principal information that highlights differences among spikes. Common spike feature extraction algorithms are based on principal component analysis (PCA) [4], the discrete wavelet transform (DWT) [5], or discrete derivatives [6]. A dimensionality reduction step may be used to select only the few best coefficients. In the final phase, spikes are assigned into different MU classes. Existing spike sorting algorithms using distances (e.g., k-means clustering [7][8]), mean shift [9][10]), likelihood (e.g., Bayesian classification (BC) [11]), or super paramagnetic clustering (SPC) [12] have all been proposed.

In the literature of MUAP discrimination, the relevance and clusterability of the above existing features have failed to be addressed. Most previous automated efforts often only yielded excellent performance for subject-dependent settings. We hypothesize that higher correlated and more separatable features across classes may improve the classification performance of unsupervised subject-independent MUAP classifiers. We propose to evaluate feature candidates using our voting-based selection approach for the MUAP sorting application. This hybrid selection scheme is a datadriven approach and can compare a comprehensive set of candidates including existing features and novel variants. The strategy has been shown successfully in detecting respiratory artefacts in lung function data [13] and freezing of gait epochs in acceleration data [14]. However, under the context of MUAP sorting, the classification involves an unknown number of class discrimination. In this work, we demonstrate that such feature analysis approach is also applicable to MUAP discrimination. The main contributions of this work are:

- This is the first reported feature analysis approach using clusterability criterion for MUAP sorting in nEMG data.

- The spectrograms of wavelet decomposition is more 
relevant and discriminative than existing features.

- The accuracy of our proposed is comparable with the manual reference.

The rest of the paper is as follows. The method details including data collection, the feature analysis process, sorting algorithms, and performance metrics are presented in Section II. The obtained results are reported and discussed in Section III and IV. Conclusion is drawn in Section V.

\section{Methods}

\section{A. Data Collection}

1) Physiologically-Based Synthetic Data: We used the nEMG simulation algorithm by Hamilton-Wright and Stashuk [15] for our development phase. Note that the algorithm was shown to produce nEMG data consistent with those acquired from real muscle (the developed muscle) [15]. We run the simulator on a Microsoft Windows personal computer for a concentric electrode during a 10\% contraction maximal voluntary $(M V C)$. Figure 1 illustrates a synthetic epoch of $100 \mathrm{~ms}$. The voltage range is $-516.05 \rightarrow$ $1019.84 \mu \mathrm{V}$. The full settings used for the experiments can be found in [15].

2) Human Recorded Data: We also collected a real data set recording from a healthy young male at the Fuglevand Laboratory [16] using a rack-mounted electro-physiological recording system CED [2]. Data were sampled at $55.5 \mathrm{kHz}$. The experiment settings for force used to create nEMG data was: time interval of $0.1 \mathrm{~ms}$ for force, scale of 0.0023 , unit of " $N$ ". We used the concentric needle electrode. A neurologist manually provided labels of MUAP appearances together with its associated MU. Note that though most of the manual labeling procedure was aided by a commercial software tool (Spike2 [2]), the human operator is still needed for the final template matching and adjusting. These labels are referred to as "reference" during our evaluation.

\section{B. Feature Analysis}

1) Selection Scheme: Given a large exploratory feature pool, a voting process with different selection levels and criteria (saliency, robustness, and accuracy) is used to figure out the best feature. After each level, selected candidates become more favourable. Specifically, the first round suggests the most salient and discriminative subset of features using mutual information (MI) and separability calculated using the Euclidean distance (DIS). These features are evaluated against the reference for detection performance assessment.

Selection criteria, i.e., MI and DIS are calculated as follows. Let $X$ be a discrete random variable $X \in \mathbb{X}$ and $C$ be a target variable $(c \in \mathbb{C}$, class label set). The entropy $H_{b}(X)$ of $X$ measures its uncertainty [17].

$$
H_{b}(X) \stackrel{\text { def }}{=}-\sum_{x \in \mathbb{X}} p(x) \log _{b} p(x)
$$

where $b$ is the base of the logarithm. In this work, $b=2$, and hence entropy will be measured in bits.

Let $C$ be a target variable $(c \in \mathbb{C}$, class label set). The conditional entropy of $X$ given $C$ is defined by:

$$
H(X \mid C)=-\sum_{c \in \mathbb{C}} p(c) \sum_{x \in \mathbb{X}} p(x \mid c) \log p(x \mid c) .
$$

The mutual information [17] between $X$ and $C, M I(X ; C)$, measures the amount of information "shared" by $X$ and $C$. MI is then interpreted as the relevance of $X$ and $C$ :

$$
I(X ; C)=\sum_{x \in \mathbb{X}} \sum_{c \in \mathbb{C}} p(x c) \log \frac{p(x c)}{p(x) p(c)}
$$

To assess discrimination of features, relevant candidates are considered having nearest instances (by Euclidean distances) of same class closer and having nearest ones of other classes more far apart. The weighting of these distances, called DIS, is calculated using the RELIEF algorithm [18] (as similarly implemented in [19] or built-in packages of MATLAB, The MathWorks Inc., Natick, MA, 2000).

2) Feature Pool: We extracted twelve groups of features in both time and frequency domains (Table I). In the table, existing features include amplitude range information of EMG data, DWT, top ten selected by KS tests [20] of DWT, top ten percentage selected by ICA or PCA. Our new feature candidates are singular value decomposition (SVD) of spectral analysis and spectrograms of raw amplitude data or DWT transformed data.

TABLE I: List of candidates in the EMG feature pool.

\begin{tabular}{|l|l|l|l|l|}
\hline $\begin{array}{l}\text { Group } \\
\text { ID }\end{array}$ & Domain & Description & $\begin{array}{l}\text { New } \\
\text { or } \\
\text { not?* }\end{array}$ & $\begin{array}{l}\text { Feature } \\
\text { ID }\end{array}$ \\
\hline 1 & Time & $\begin{array}{l}\text { Maximum amplitude of } \\
\text { EMG }\end{array}$ & No & 1 \\
\hline 2 & Time & $\begin{array}{l}\text { Minimum amplitude of } \\
\text { EMG }\end{array}$ & No & 2 \\
\hline 3 & Time & $\begin{array}{l}\text { Range amplitude of } \\
\text { EMG }\end{array}$ & No & 3 \\
\hline 4 & Frequency & DWT level d3 & No & $4-128$ \\
\hline 5 & Frequency & DWT level d4 & No & $129-253$ \\
\hline 6 & Frequency & DWT level a3 & No & $254-378$ \\
\hline 7 & Frequency & $\begin{array}{l}\text { SVD of spectral analy- } \\
\text { sis }\end{array}$ & Yes & $379-386$ \\
\hline 8 & Time & ICA (ten percentage) & No & $387-398$ \\
\hline 9 & Time & PCA (ten percentage) & No & $399-410$ \\
\hline 10 & Frequency & $\begin{array}{l}\text { KS test of DWT (top ten } \\
\text { coefficients) }\end{array}$ & No & $411-420$ \\
\hline 11 & Frequency & $\begin{array}{l}\text { Spectrograms of raw } \\
\text { amplitude }\end{array}$ & Yes & $\begin{array}{l}421- \\
1065\end{array}$ \\
\hline 12 & Frequency & $\begin{array}{l}\text { Spectrograms of DWT } \\
\text { Yes }\end{array}$ & $\begin{array}{l}1066- \\
1710\end{array}$ \\
\hline
\end{tabular}

Several methods used for the new feature extraction (i.e., they have not been proposed for nEMG spike sorting) are described as follows. Discrete wavelet analysis that represents signals in both frequency and time is a very useful tool in the neuroscience field [21]. Transient differences in high frequency features (sharp edges and steep leading or trailing slopes) and/or in low frequency features (duration of the repolarization phase) can present the morphology of spikes. In this work, MUAPs are first decomposed into wavelet coefficients using the DWT method [5]. These coefficients represent differences among spikes based on the quantification of energy found in specific frequency bands at specific time locations. We implemented a 4-level decomposition and Haar window using built-in functions of MATLAB (The MathWorks Inc., Natick, MA, 2000).

Due to the multi-modal distribution of coefficients [22], we rank these candidates by scores calculated by deviation from normality, using a modification of Kolmogorov- 


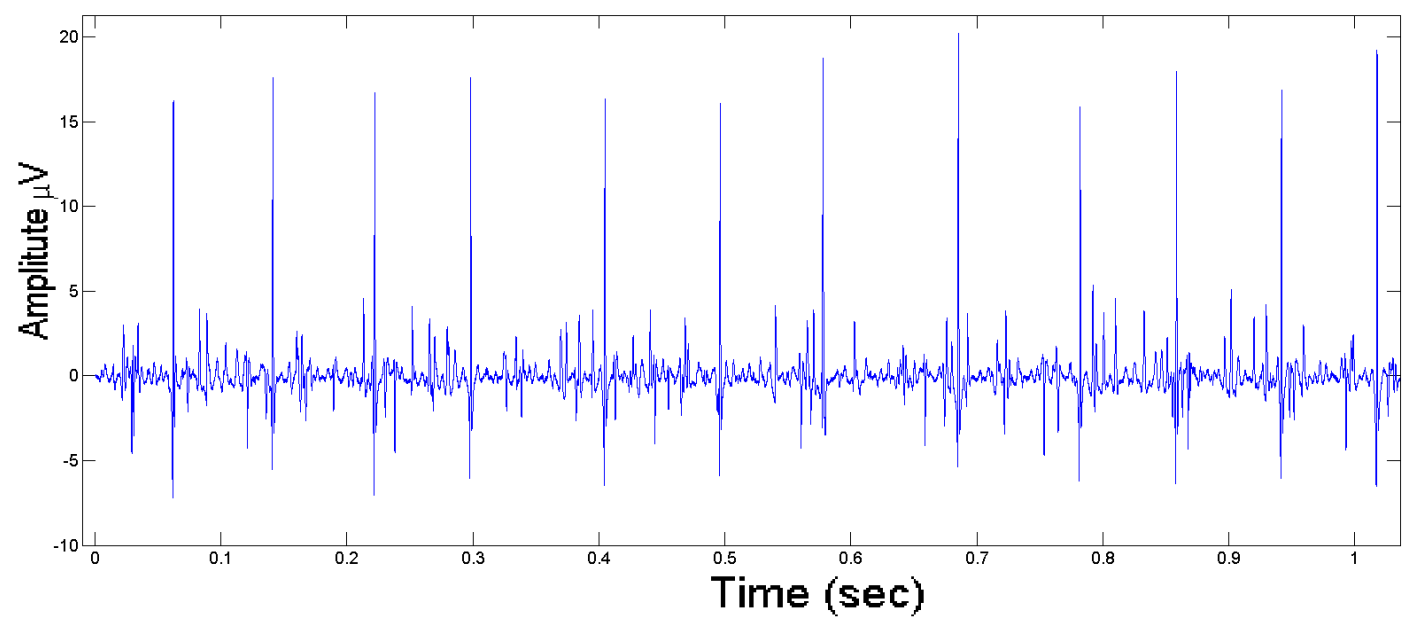

Fig. 1: Example of a 100-ms epoch of the simulated nEMG.

Smirnov (KS) test [20]. Let $X$ be a data set, the score is $\max (|F(x) G(x)|)$ where $F(x)$ is the cumulative distribution function of $X$ and $G(x)$ is a Gaussian cumulative distribution function with the same mean and variance. To minimize the effect of overlapping spikes, for each coefficient, only values within three standard deviations (both directions) are considered [22]. In order to create a compressed input to the sorting process, only the ten largest score candidates are selected to best separate spikes.

Finally, these selected coefficients are transformed to a series of spectral snapshots (spectrograms) using the short Fourier transform (STFT [23]). Specifically, let $v$ be the wavelet feature of a spike. A Hamming window is used with STFT to transform $v$ into an image of spectrogram. Hence, distance between spikes are the correlation coefficients between these images.

\section{Automated Spike Sorter}

1) Preprocessing: Intramuscular data is corrupted by spike-like correlated noise. Thus, we need to make data points statistically independent ("pre-whitening"). A practical approach employs a linear prediction filter [24] to whiten the input signal itself before we extract any MUAP. In this work, we use a third-order forward linear predictor (FIR filter) that predicts the current value of the real-valued original data based on past three samples [24]. Using timing labels from the reference, we extract the spike set together with labels of MU classes. All spikes are extracted with the same window size of $8 \mathrm{~ms}$.

To focus on sorting evaluation, overlapping spikes (i.e., have more than two MU in the same window) relate more to spike detection than sorting algorithms. Thus, we removed overlapping spikes with small delay by detecting multiple peaks within a spike window. For overlaps without delay (i.e., they may look like the firing of a new neuron), we do a re-sorting step as described in the post-preprocessing section.

2) $x$-Class Sorter: After feature extraction steps, based on MUAP morphology, the correlation between spikes is used as the similarity measure for an number x-class sorting application [25], [26] where $x$ is unknown. Instead of using the Euclidean distance metric, to account for electrode drift and normalized values that suit for subject-independent settings, we proposed to use the correlation metric that ranges from 0 to 1 .

Let $I_{X}$ and $I_{Y}$ be two feature vectors of MUAP $X$ and MUAP $Y$, respectively. $r_{X, Y}$ is the correlation between two feature vectors of $X$ and $Y \quad r_{X, Y}=\frac{\mathscr{C}\{X, Y\}}{\sigma_{X} \sigma_{Y}}$ where $r_{X, Y}$ is the correlation coefficient between MUAP $X$ and MUAP $Y$. $\mathscr{C}\{X, Y\}$ is the covariance of two feature vectors $X$ and $Y . \sigma_{X}$ and $\sigma_{Y}$ are the variances of $X$ and $Y$, respectively. The class assignment variable of $X$ is defined by the correlation based sorting scheme. The sorter starts with a single class contains all spikes having high correlation $r_{X, Y}$ with the initial spike given a desired threshold level (e.g., 0.9). Then the sorter stops when the unsorted pool of remaining spikes is empty.

3) Post-processing: Because the firing behaviour of an individual MU relates to its recruitment threshold [27] [28], the size of a valid cluster corresponding to a MU should exceed a parameter. According to the recruitment threshold assignment derived from the work of Fuglevand [29]) and popular settings found in the literature, we set this parameter to 40. All clusters with size smaller than 40 were merged into a group, called catch-all class. We assume that these small clusters may associate with overlapping spikes without delay. To assign the labels for clusters (or individual spike if that is the catch-all cluster), we measure the correlation between the mean waveform of the cluster and the one of the reference group. A label is chosen if the match has the highest correlation score.

\section{Reference Works}

The objective reference clustering results are available for the synthetic data as the simulator is controlled during data generation. However, this is usually not available for the recorded data. Ideally the reference could be derived from simultaneous intra-cellular recording, but availability of such data is limited. The most common practice in physiology laboratories involves using commercial software (e.g., Spike2 in our work) and manual checking by an (human) operator. This approach was used to obtain the reference in this work for real recordings. 
We also compare our proposed method with a relevant work using the DWT extraction and super paramagnetic clustering (SPC) [12]. We applied settings for the SPC method as recommended in [30]. Specifically there were $q=20$ states, $K=11$ nearest neighbours, and $N=500$ iterations for clustering. The range of temperature was from 0 to 0.201 in steps of 0.01 . The implementation was provided by the authors of [12] (MATLAB packages, The MathWorks Inc., Natick, MA, 2000) .

\section{E. Performance Metrics}

Performance metrics for a multi-class classification task are derived from the confusion matrix. Let $M$ be the confusion matrix of sorting outcome. The successful predicted events (True) for a class are on the diagonal of $M$. All other members of $M$ are incorrectly predicted events (False). Let $M_{i j}$ denote the number of test outcomes (i.e., ground truth labels, Ground ${ }_{i}$ ) of class $i$, that were predicted as class $j$, Predicted $_{j}$. The successful predicted events (True) for class $i$, denoted $T_{i i}$, are on the diagonal of $M$. All other members of $M$ are incorrectly predicted events (False), denoted $F_{i j}$ where $i \neq j$.

$M=\left(\begin{array}{cccccc}\text { Predicted }_{1} & \ldots & \text { Predicted }_{i} & \ldots & \text { Predicted }_{C} & \\ T_{11} & \ldots & F_{1 i} & \ldots & F_{1 C} & \text { Ground }_{1} \\ \vdots & \ddots & \vdots & \ldots & \vdots & \vdots \\ F_{i 1} & \ldots & T_{i i} & \ldots & F_{i C} & \text { Ground }_{i} \\ \vdots & \ldots & \vdots & \ddots & \vdots \\ F_{C 1} & \ldots & F_{C i} & \ldots & T_{C C}\end{array}\right)$ Ground $_{C}$

The sensitivity and positive predictive value (PPV) of class $i, \operatorname{Sen}_{i}$ and $\mathrm{PPV}_{i}$, are defined as follows.

$$
\begin{aligned}
\operatorname{Sen}_{i} & =\frac{T_{i i}}{T_{i i}+\sum_{j \neq i} F_{i j}} \\
\operatorname{PPV}_{i} & =\frac{T_{i i}}{T_{i i}+\sum_{j \neq i} F_{j i}}
\end{aligned}
$$

\section{RESULTS}

\section{A. Selective Features}

Ranking over the entire exploratory pool, each feature candidate was found with a ranking score by aforementioned saliency criteria (Fig. 2; sorted from high to low scores. The higher saliency score indicated the higher ranking order. Among the top quarter (i.e., highest $25 \%$ ranking) of the pool, the distribution of feature groups (Fig. 2b) shows that the list includes DWT and Spectrograms of DWT coefficient features (Table I).

As can be seen, scores dropped quickly outside of the top 25 percent candidates by DIS criterion and only after 80 percent candidates by the MI score. We found that, by MI criterion, except for the single feature of Group 1, all other members of the top 25 percent belong to Group 12 . Meanwhile, by DIS criterion, though the top 25 percent includes several groups, Group 12 still dominates the high score area. Hence, we proposed to use the feature set Group 12 for the next evaluation in terms of sorting performance.

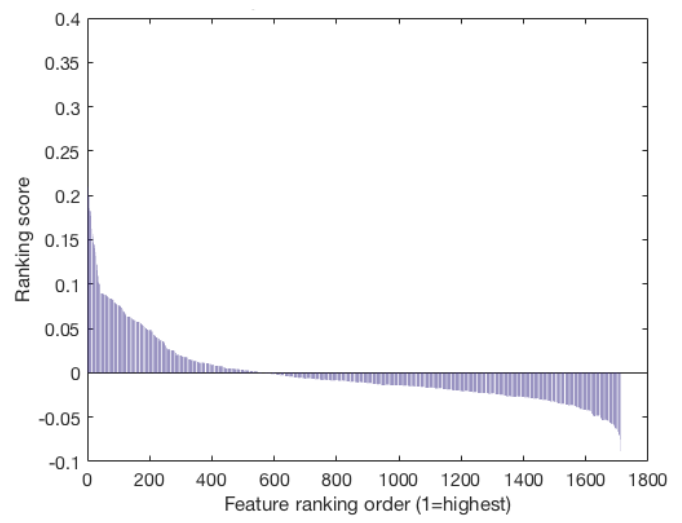

(a) Ranking of the entire feature pool.

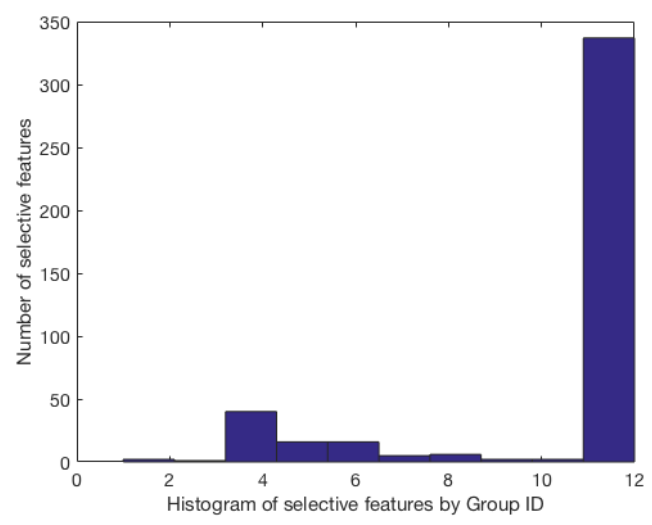

(b) The histogram of the top 25 percent candidates.

Fig. 2: Example of feature ranking by DIS criterion. (a) Ranking scores for the entire feature pool. Vertical: saliency scores; Horizontal: ranking order (highest $=1$, lowest $=1710$ ). (b) The histogram of the top 25 percent highest-score candidates by feature groups (Table I).

\section{B. Sorting Performance}

1) Synthetic Data: After preprocessing, spike sets were prepared for the sorting stage as in Table II. In the reference set, the MU1 class has much larger amplitude range than other four classes. Classes MU2-5 have only slight difference in the waveforms. Our sorter produced five clusters that match with five reference classes. After assigning labels, the histograms were compared with the reference histogram (Fig. 3). In terms of the confusion matrix, the general classification accuracy and class-wise sensitivities as well as predictivities are reported in Table III.

TABLE II: Spike set inputs. Class proportions are in order of the MU names in the labels.

\begin{tabular}{lll}
\hline & Synthetic data & Recorded data \\
\hline Number of spikes & 1230 & 1220 \\
\hline Number of classes & 5 & 3 \\
\hline Class proportion & $336: 269: 226: 207: 192$ & $440: 483: 535$ \\
\hline
\end{tabular}

2) Recorded Data: Table IV depicts the distribution of spikes in large clusters corresponding to the reference classes from the recorded dataset. Both automatic clustering methods had about $19 \%$ catch-all spikes. There were three reference classes. While the amplitude range of spikes in MU1 and 
Reference

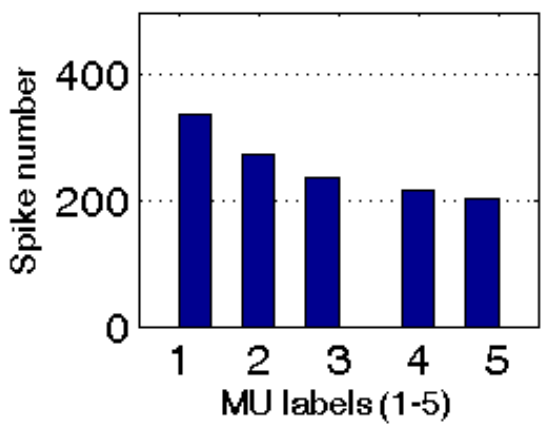

Compared

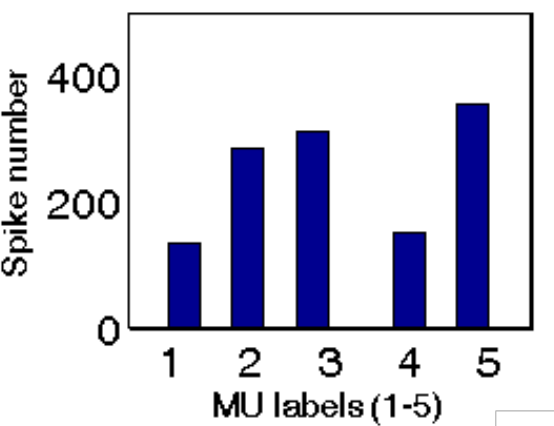

Proposed

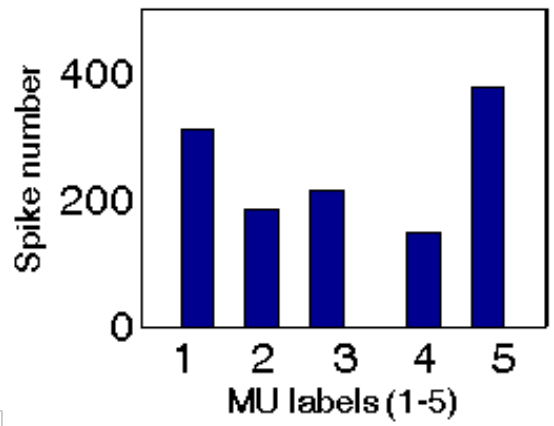

Fig. 3: Agreement in histograms of automatic methods against the reference for synthetic data.

TABLE III: Synthetic data MUAP sorting comparisons between automatic methods and the reference. Accuracy measures (in \%) use simulation settings as reference. True/False are MU matching or not with the reference labels.

\begin{tabular}{|c|c|l|l|l|l|}
\hline \multirow{3}{*}{ Metrics } & \multirow{2}{*}{ Class name } & \multicolumn{2}{|c|}{ Include catch-all } & \multicolumn{2}{l|}{ Not include catch-all } \\
\cline { 3 - 6 } & $\begin{array}{l}\text { SPC- } \\
\text { based }\end{array}$ & $\begin{array}{l}\text { Our } \\
\text { method }\end{array}$ & $\begin{array}{l}\text { SPC- } \\
\text { based }\end{array}$ & $\begin{array}{l}\text { Our } \\
\text { method }\end{array}$ \\
\hline \multirow{4}{*}{ Sensitivity } & MU1 & 39.3 & 92.5 & 100.0 & 100.0 \\
\cline { 2 - 6 } & MU2 & 85.1 & 67.6 & 100.0 & 98.4 \\
\cline { 2 - 6 } & MU3 & 74.7 & 75.2 & 98.8 & 98.3 \\
\cline { 2 - 6 } & MU4 & 71.9 & 69.5 & 99.3 & 88.9 \\
\cline { 2 - 6 } & MU5 & 19.3 & 88.0 & 0 & 72.9 \\
\hline \multirow{4}{*}{ PPV } & MU1 & 100.0 & 100.0 & 100.0 & 100.0 \\
\cline { 2 - 6 } & MU2 & 81.2 & 98.9 & 98.2 & 98.9 \\
\cline { 2 - 6 } & MU3 & 54.5 & 80.2 & 54.5 & 80.2 \\
\cline { 2 - 6 } & MU4 & 98.6 & 97.9 & 54.6 & 97.9 \\
\cline { 2 - 6 } & MU5 & 10.4 & 44.9 & 0 & 100.0 \\
\hline \multicolumn{2}{|c|}{ Average accuracy } & 58.2 & 79.3 & 81.9 & 94.8 \\
\hline
\end{tabular}

MU2 are $\pm 0.5 \mu \mathrm{V}$, MU3 ranges much larger $( \pm 1 \mu \mathrm{V})$. Sorting performance for each MU and the general accuracy were depicted in Table V.

TABLE IV: Distribution of spike count in large clusters corresponding to the reference classes from the recorded dataset.

\begin{tabular}{|l|c|c|c|}
\hline Clusters & Our method & SPC-based & Manual reference \\
\hline MU1 MUAPs & 314 & 368 & 383 \\
\hline MU2 MUAPs & 283 & 335 & 408 \\
\hline MU3 MUAPs & 378 & 284 & 429 \\
\hline un-sorted MUAPs & 245 & 233 & 0 \\
\hline
\end{tabular}

In contrast with our superior results against the SPC when applied to the synthetic data, results of both automatic techniques were comparable with recorded data. However, it might be due to the small size of dataset and a small number of active MUs recorded. We may also need an interrater measurement to alleviate the human subjectivity of the manual reference in evaluation. These should be addressed in future work for the method. In general, all performance measurements we achieved in this study are among the most accurate outcomes in spike sorting evaluation works.

\section{DISCUSSION}

Given a large exploratory feature pool, to select the best feature, a voting process consists of three levels: saliency,
TABLE V: Comparison of sorting performance using recorded data between automatic methods. Accuracy measures (in \%) use manual labels as reference. True/False are MU matching or not with the reference labels.

\begin{tabular}{|c|c|l|l|l|l|}
\hline \multirow{3}{*}{ Metrics } & \multirow{2}{*}{ Class name } & \multicolumn{2}{|c|}{ Include catch-all } & \multicolumn{2}{l|}{ Not include catch-all } \\
\cline { 3 - 6 } & $\begin{array}{l}\text { SPC- } \\
\text { based }\end{array}$ & $\begin{array}{l}\text { Our } \\
\text { method }\end{array}$ & $\begin{array}{l}\text { SPC- } \\
\text { based }\end{array}$ & $\begin{array}{l}\text { Our } \\
\text { method }\end{array}$ \\
\hline \multirow{3}{*}{ Sensitivity } & MU1 & 99.7 & 99.7 & 99.7 & 99.6 \\
\cline { 2 - 6 } & MU2 & 89.9 & 68.6 & 100.0 & 99.6 \\
\cline { 2 - 6 } & MU3 & 74.6 & 83.9 & 100.0 & 100.0 \\
\hline \multirow{3}{*}{ PPV } & MU1 & 71.8 & 65.8 & 100.0 & 99.6 \\
\cline { 2 - 6 } & MU2 & 99.7 & 99.6 & 99.7 & 99.6 \\
\cline { 2 - 6 } & MU3 & 100.0 & 100.0 & 100.0 & 100.0 \\
\hline \multicolumn{2}{|c|}{ General accuracy } & 87.6 & 83.7 & 99.9 & 99.8 \\
\hline
\end{tabular}

robustness, and accuracy selection. This strategy has been successfully demonstrated with anomaly detection scenarios in our earlier works [13], [14]. However, both of the cases are two-predefined-class detection tasks. Though MUAP sorting involves an unknown-class-number classification task, the feature analysis scheme still addresses the best feature candidate for the clustering purpose. Synthetic and human recorded datasets of motor unit action potentials were used to demonstrate the performance. Compared with the manual reference, our MUAP sorting method is comparable (regarding to the number of MUs found and histograms of MUs). Moreover, in the compared method (SPC-based), the temperature terminology used for reviewing outcome is less intiuitive than the correlation as in our method. The correlation values range $0 \rightarrow 1$ while the measure of temperature is difficult to tune.

\section{Conclusion}

In this work, a feature analysis approach for spike sorting in MUAP is reported. We analyzed a large pool of candidates for MUAP feature extraction. We used ranking scores by several saliency criteria including mutual information, Euclidean distance based discrimination. This hybrid selection scheme is a data-driven approach and can compare a comprehensive set of candidates including existing features and novel variants. The most selective features learnt from this process are most applicable to the unsupervised and subject-independent applications. We demonstrated the classification performance with this feature using both synthetic nEMG and human recorded data. Compared with the reference produced by 

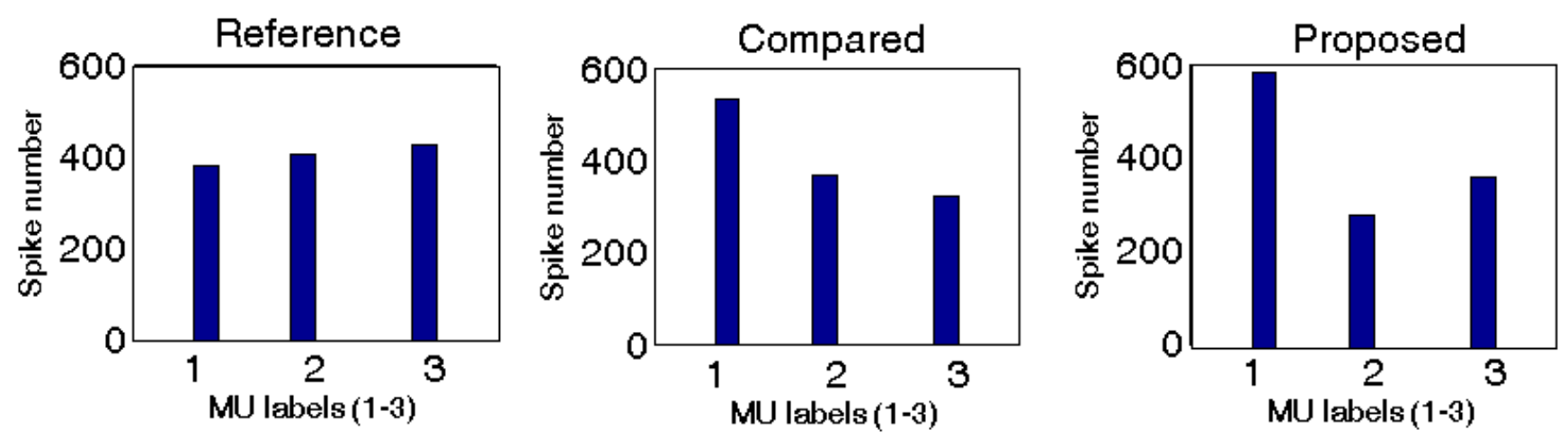

Fig. 4: Agreement in histograms of automatic methods against the reference for recorded data.

human experts, our method obtained a comparable result (e.g., equivalent number of classes was found, identical MUAP morphology in each pair of corresponding MU class, and similar histograms of MUs).

\section{REFERENCES}

[1] S. Paganoni and A. Amato, "Electrodiagnostic evaluation of myopathies," Physical medicine and rehabilitation clinics of North America, vol. 24, no. 1, pp. 193-207, 2013.

[2] Cambridge Electronic Design: Spike2, www.ced.co.uk.

[3] Cerebus, www.cyberkineticsinc.com.

[4] E. M. Glaser and W. B. Marks, "Separation of neuronal activity by waveform analysis," Advances in Biomedical Engineering, vol. 5, pp. 137-156, 1968

[5] J. C. Letelier and P. P. Weber, "Spike sorting based on discrete wavelet transform coefficients," Journal of Neuroscience Methods, vol. 101, no. 2, pp. 93 - 106, 2000.

[6] V. Karkare, S. Gibson, and D. Markovic, "A $130 \mu W$, 64-channel spike-sorting DSP chip," in Solid-State Circuits Conference, 2009. ASSCC 2009. IEEE Asian, 2009, pp. 289 -292.

[7] S. P. Lloyd, "Least squares quantization in pcm," Information Theory, IEEE Transactions on, vol. 28, no. 2, pp. 129-137, 1982.

[8] S. Takahashi, Y. Anzai, and Y. Sakurai, "A new approach to spike sorting for multi-neuronal activities recorded with a tetrode-how ICA can be practical," Neuroscience Research, vol. 46, no. 3, pp. $265-$ 272, 2003.

[9] Y. Cheng, "Mean shift, mode seeking, and clustering," Pattern Analysis and Machine Intelligence, IEEE Transactions on, vol. 17, no. 8, pp. 790-799, 1995.

[10] Z. Yang, Q. Zhao, and W. Liu, "Improving spike separation using waveform derivatives," Journal of Neural Engineering, vol. 6, pp. 046 006-046018, 2009.

[11] P. Cheeseman, J. Kelly, M. Self, J. Stutz, W. Taylor, and D. Freeman, "Autoclass: A Bayesian classification system," Proc of the Fifth Intl Workshop on Machine Learning, pp. 54-64, 1988.

[12] M. Blatt, S. Wiseman, and E. Domany, "Superparamagnetic clustering of data," Phys. Rev. Lett., vol. 76, pp. 3251-3254, 1996.

[13] T. T. Pham, S. T. Moore, S. J. Lewis, D. N. Nguyen, E. Dutkiewicz, A. J. Fuglevand, A. L. McEwan, and P. H. Leong, "Freezing of gait detection in parkinsons disease: A subject-independent detector using anomaly scores," IEEE Transactions on Biomedical Engineering, 2017.

[14] T. T. Pham, C. Thamrin, P. D. Robinson, A. McEwan, and P. H. Leong, "Respiratory artefact removal in forced oscillation measurements: A machine learning approach," IEEE Transactions on Biomedical Engineering, 2016.

[15] A. Hamilton-Wright and D. W. Stashuk, "Physiologically based simulation of clinical emg signals," IEEE Transactions on biomedical engineering, vol. 52, no. 2, pp. 171-183, 2005.

[16] Fuglevand Laboratory of motor control neurophysiology, Department of Physiology, University of Arizona, USA.

[17] C. Shannon, "A mathematical theory of communication," Bell System Technical Journal, The, vol. 27, no. 3, pp. 379-423, July 1948.

[18] K. Kira and L. A. Rendell, "The feature selection problem: traditional methods and a new algorithm," in Proceedings of the tenth national conference on Artificial intelligence, ser. AAAI'92. AAAI Press, 1992, pp. 129-134.
[19] G. Brown, A. Pocock, M.-J. Zhao, and M. Luján, "Conditional likelihood maximisation: a unifying framework for information theoretic feature selection," The Journal of Machine Learning Research, vol. 13, no. 1, pp. 27-66, 2012.

[20] H. W. Lilliefors, "On the kolmogorov-smirnov test for normality with mean and variance unknown," Journal of the American Statistical Association, vol. 62, no. 318, pp. 399-402, 1967.

[21] V. J. Samar, "Wavelet analysis of neuroelectric waveforms," Brain and Language, vol. 66, pp. 1 - 6, 1999.

[22] R. Quiroga, Z. Nadasdy, and Y. Ben-Shaul, "Unsupervised spike detection and sorting with wavelets and superparamagnetic clustering," Neural Comput., vol. 16, pp. 1661-1687, 2004.

[23] D. Gabor, "Theory of communication. part 1: The analysis of information," Electrical Engineers-Part III: Radio and Communication Engineering, Journal of the Institution of, pp. 429-441, 1946.

[24] L. B. Jackson et al., Digital filters and signal processing. Springer, 1989, vol. 3.

[25] T. T. Pham, A. J. Fuglevand, A. L. McEwan, and P. H. Leong, "Unsupervised discrimination of motor unit action potentials using spectrograms," in Engineering in Medicine and Biology Society $(E M B C), 2014$ 36th Annual International Conference of the IEEE. IEEE, 2014, pp. 1-4.

[26] T. T. Pham and C. M. Higgins, "A visual motion detecting module for dragonfly-controlled robots," in Engineering in Medicine and Biology Society (EMBC), 2014 36th Annual International Conference of the IEEE. IEEE, 2014, pp. 1666-1669.

[27] J. V. Basmajian and C. De Luca, "Muscles alive," Muscles alive: their functions revealed by electromyography, vol. 278, p. 126, 1985.

[28] C. J. De Luca, "Physiology and mathematics of myoelectric signals," IEEE Transactions on Biomedical Engineering, no. 6, pp. 313-325, 1979.

[29] A. J. Fuglevand, D. A. Winter, and A. E. Patla, "Models of recruitment and rate coding organization in motor-unit pools," Journal of neurophysiology, vol. 70, no. 6, pp. 2470-2488, 1993.

[30] M. Blatt, S. Wiseman, and E. Domany, "Data clustering using a model granular magnet," Neural Comput., vol. 9, pp. 1805-1842, 1997. 